Original

\title{
Histopathological Changes of Streptozotocin-induced Painful Diabetes and Antihyperalgesic Effect of Capsaicin Cream in Rats
}

\author{
Osamu Mitani ${ }^{1}$, Kuniharu Masui ${ }^{1}$, Hisashi Tsujimoto ${ }^{1}$, Keisuke Jinbo ${ }^{1}$, \\ Yukihiko Watanabe ${ }^{1}$, Takako Ohkura ${ }^{1}, K_{\text {Koji Taya }}{ }^{1}$, and Hitoshi Ikeda ${ }^{1}$ \\ ${ }^{1}$ Central Research Laboratory, Maruishi Pharmaceutical Co., Ltd., 2-2-18 Imazu-naka, Tsurumi-ku, Osaka, 538-0042 \\ Japan
}

\begin{abstract}
To investigate the association between streptozotocin (STZ)-induced painful diabetic neuropathy and the antihyperalgesic effect of capsaicin cream in rats, we first examined the antihyperalgesic effect of capsaicin cream and subsequently performed peripheral neurohistochemical examinations of neuropathic rats. Mechanical hyperalgesia occurred 2 weeks after STZ injection and persisted for 8 weeks of testing. The neuropathy was alleviated by a single application of $0.1 \%$ capsaicin cream, but not by cream base. The hyperalgesia did not decrease when the capsaicin cream was applied to normal animals. On the other hand, for dorsal root ganglion (DRG) neurons and hind paw cutaneous nerves, the neurohistochemical examination showed no difference between STZ rats and control rats with capsaicin (vanilloid) receptor subtype 1 (VR1) and NF200 double immunohistochemical staining of DRG neurons, but an increase in A-fibers was seen in the hind paw cutaneous nerves of the STZ rats compared to the control rats. Neither STZ rats nor control rats were toxically affected by the single application of $0.1 \%$ capsaicin cream. These results suggest that a single application of capsaicin cream exerts an antihyperalgesic effect in rats with painful neuropathy and increases peripheral A-fibers. However, we could not clarify how VR1 was involved in the effect in rats with STZ painful diabetic neuropathy. (J Toxicol Pathol 2008; 21: 97-104)
\end{abstract}

Key words: streptozotocin, diabetic neuropathy, capsaicin, VR1

\section{Introduction}

In rats, a single systemic injection of streptozotocin (STZ) induces short-term insulin-deficient diabetes ${ }^{1,2}$. STZ is an antibiotic extracted from Streptomyces acromogenes and is diabetogenic due to its selective cytotoxic action on pancreatic beta cells ${ }^{1}$. STZ-induced diabetic rats have been increasingly used as a model of painful diabetic neuropathy to assess the efficacies of potential analgesic agents ${ }^{3-6}$. However, the underlying mechanisms of the development of diabetic neuropathy are poorly understood. Hyperglycemia is considered to be a major pathogenic factor in the development of diabetic neuropathy.

Capsaicin, [(E)-N-(4-hydroxy-3-methoxybenzyl)-8methyl-6-onenamide], a pungent ingredient of chili peppers, is widely used in clinical settings to alleviate various painful conditions, including diabetic neuropathic pain ${ }^{7,8}$. Capsaicin

Received: 16 January 2008, Accepted: 25 February 2008

Mailing address: Osamu Mitani, Central Research Laboratory,

Maruishi Pharmaceutical Co., Ltd., 2-2-18 Imazu-naka, Tsurumi-ku,

Osaka, 538-0042, Japan

TEL: 81-6-6962-1784 FAX: 81-6-6962-2948

E-mail: osamu_mitani@maruishi-pharm.co.jp is known to be an algesic substance and has long been used as an experimental tool because of its rather selective action on small-diameter afferent nerve fibers (C-fibers). Capsaicin stimulates the vanilloid receptor subtype 1 (VR1) located mainly on polymodal C-fibers and initiates a complex cascade of events, including neuronal excitation and release of proinflammatory mediators, desensitization of the receptor, and neuronal toxicity ${ }^{9}$. It can be considered a molecular integrator of noxious chemical and heat stimuli in the peripheral terminals of polymodal nociceptors ${ }^{10-12}$.

According to a previous study ${ }^{13}$, the application of capsaicin cream dose- dependently reversed the thermal, mechanical, and chemical hyperalgesia observed in STZinduced diabetic mice. Moreover, the study confirmed the up-regulation of VR1 expression on myelinated primary afferent neurons of diabetic mice by immunohistochemistry. The study results suggest that increased expression of VR1 on myelinated fibers might contribute to the antihyperalgesic effect of topical capsaicin on diabetic neuropathic pain. The underlying mechanism of the effect is regarded as desensitization of VR1. However, some reports suggest that topical application of capsaicin in humans results in the degeneration of epidermal nerve fibers with a corresponding 
decrease in detection of heat pain sensation ${ }^{14,15}$. This degeneration is a reversible change, but it is considered that the degeneration is due to toxic action rather than the pharmacological action of capsaicin. Although it is now considered that the antihyperalgesic effect of capsaicin is due to desensitization of VR1, it is not well understood how the desensitization of VR1 and histologic change of the nervous system are related.

In the present study, we identified the antihyperalgesic effect of capsaicin in animal models of painful diabetic neuropathy and normal animals, removed the area of the skin to which capsaicin was applied and the dorsal root ganglion (DRG) innervated nerves of the area from all animals, and immunohistochemically examined changes in those tissues.

\section{Materials and Methods}

\section{Animals}

Six-week-old Sprague-Dawley male rats (Japan SLC, Inc., Hamamatsu, Japan) weighing 184 to $221 \mathrm{~g}$ were used for this study. The animals were kept under standardized conditions (12-hr light/dark cycle, 40 to $70 \%$ relative humidity, 20 to $24^{\circ} \mathrm{C}$ room temperature) at Central Research Laboratory, Maruishi Pharmaceutical Co., Ltd. with free access to water and standardized food.

This study was reviewed and approved by the Animal Ethics Committee of Maruishi Pharmaceutical Co., Ltd.

\section{Drugs}

The capsaicin cream, labeled $0.1 \%$, contained $1 \mathrm{mg}$ of capsaicin in $1 \mathrm{~g}$ of hydrophilic cream base. The cream base contained polyoxyethylated castor oil, liquid paraffin, white petroleum jelly, 1-hexadecanol, EDTA disodium salt, and triethanolamine.

\section{STZ-induced diabetes}

Diabetes was induced in normal rats by a single intravenous tail vein injection of $50 \mathrm{mg} / \mathrm{kg} \mathrm{STZ}$ (Wako Pure Chemical Industries, Ltd., Osaka, Japan) dissolved in sodium citrate $(0.1 \mathrm{~N}, \mathrm{pH} 4.5)^{16}$. Age-matched non-diabetic control rats were injected with vehicle alone. One week after the STZ or vehicle injection, the blood glucose level in the rats was measured using a blood glucose meter (Sanwa Kagaku Kenkyusho Co., Ltd., Nagoya, Japan) in blood samples obtained from their tail veins.

Rats with plasma glucose levels above $400 \mathrm{mg} / \mathrm{dL}$ were considered diabetic.

\section{Nociceptive test}

In assessing the antihyperalgesic effect of capsaicin cream in animal models of painful diabetic neuropathy and normal animals, we used a Randall-Sellito paw pressure device to study hyperalgesia that developed in STZ-diabetic rats $^{3,4,6}$. The mechanical nociceptive threshold was quantified using an Analgesy-Meter (Ugo Basile, Varese, Italy). This instrument generates a mechanical force that increases linearly with time. The force is applied directly to the dorsal surface of the rat via a dome-shaped plunger. The nociceptive threshold is defined as the force, in grams, at which the rat attempts to withdraw both its hind paws (each paw, cut-off force of $500 \mathrm{~g}$ ).

\section{Experimental design}

After 1, 2, 4, and 6 weeks of diabetes, we performed a nociceptive test and confirmed that STZ diabetes (STZ group, $\mathrm{n}=6$ ) induced hyperalgesia, whereas vehicle injection (control group, $\mathrm{n}=6$ ) did not. STZ hyperalgesia occurred 2 weeks after STZ injection and persisted for 6 weeks. This hyperalgesia did not appear to be accompanied by acute changes in blood glucose level because the level was elevated as soon as 3 days after the STZ injection, whereas no change was present in the nociceptive threshold at this time point ${ }^{4}$. After 7 weeks of diabetes, a nociceptive test was performed, and then $100 \mathrm{mg}$ of cream base was applied to the rat footpad skin, which was kept in that state for 3 hours by covering with adhesive tape to prevent the rat from licking off the cream. After 3 hours, the applied cream base was cleanly removed, and a nociceptive test was performed again. Similarly, after 8 weeks of diabetes, a nociceptive test was performed to investigate the effect of the $0.1 \%$ capsaicin cream.

\section{Histochemical examination}

For histological examination, control rats and diabetic rats were used. The rats were deeply anesthetized with diethyl ether and sacrificed by decapitation. Subsequently, the bilateral footpad skin and DRGs of the 4th, 5th and 6th lumbar segments (L4, 5, and 6) were removed, because L4, 5 , and 6 DRGs innervate the hindlimbs ${ }^{17}$. The removed tissues were kept fixed in cold acetone for 30 minutes by the AMeX method and then embedded in paraffin ${ }^{18}$. In addition, the pancreas was removed and fixed in $10 \%$ neutral buffered formalin and then embedded in paraffin. All sections of 3 $\mu \mathrm{m}$ thickness were stained with hematoxylin and eosin (HE).

\section{Immunohistochemical examination}

For the pancreases, sections of $4 \mu \mathrm{m}$ thickness were stained by the indirect immunofluorescence technique. Primary antibodies included mouse anti-human insulin (Nichirei Corporation, Tokyo, Japan). Secondary antibodies included a combination of amino acid polymers and peroxidase-conjugated goat anti-mouse Fab fragments (Histofine $^{\circledR}$ Simple Stain Rat MAX PO (M), Nichirei Corporation, Japan). Then, the sections were visualized using diaminobenzidine. The specimens were examined under a bright-field microscope (Olympus Corporation, Tokyo, Japan).

For DRGs, sections of $4 \mu \mathrm{m}$ thickness were stained by the indirect double immunofluorescence technique. The primary antibodies were rabbit anti-rat vanilloid receptor 1 (VR1; Affinity BioReagents, Inc., Golden, Colorado, USA), and mouse anti-NF200 (N52 clone; Sigma-Aldrich, St. Louis, Missouri, USA). The secondary antibodies were donkey anti-rabbit Alexa Fluor 488, and goat anti-mouse 
Alexa Fluor 555 (Invitrogen Corporation, Carlsbad, California, USA). The sections were mounted using mounting medium with 4',6-diamidino-2-phenylindole (VECTASHIELD Mounting Medium with DAPI; Vector Laboratories, Burlingame, California, USA) and examined under a fluorescence microscope (Olympus Corporation, Tokyo, Japan). For the evaluation of VR1 and NF200 expression, the respective number of expression cells were counted in DRG cells, and the positive rate was calculated.

For the hind paw skin, sections of $30 \mu \mathrm{m}$ thickness were stained by the indirect double immunofluorescence technique. The primary antibodies were rabbit anti-human Protein Gene Product 9.5 (PGP9.5; Ultraclone Limited, Wellow, Isle of Wight, UK), and mouse anti-NF200 (N52 clone; Sigma-Aldrich, St. Louis, Missouri, USA). The secondary antibodies were donkey anti-rabbit Alexa Fluor 488, and goat anti-mouse Alexa Fluor 555 (Invitrogen Corporation, Carlsbad, California, USA). The sections were mounted using mounting medium with DAPI (VECTASHIELD Mounting Medium with DAPI; Vector Laboratories, Burlingame, California, USA) and examined under a laser-scanning confocal microscope (LSM 510 META; Carl Zeiss MicroImaging GmbH, Göttingen, Germany). Confocal images were acquired at $1-\mu \mathrm{m}$ intervals throughout the depth of the specimen. Each image could be viewed individually or the entire z-series could be projected into a single focused image. For the evaluation of PGP9.5 and NF200 expression, and each expressed area in the epidermal and dermal layer was calculated semiquantitatively by image analyzing software (LuminaVision, Mitani Corporation, Tokyo, Japan).

\section{Statistical analysis}

All data were expressed as mean \pm standard deviation (S.D.). Significance was analyzed by Student's $t$-test using SAS System Release 8.2 (SAS Preclinical Package, Version 5.0, SAS Institute Japan Ltd., Tokyo, Japan). It was considered that $\mathrm{P}$ values lower than 0.05 indicated statistical significance.

\section{Results}

\section{General results in STZ-induced diabetic rats}

Diabetes was induced in rats by intravenous injection of STZ. Diabetic rats suffered from the usual symptoms of the model of painful diabetic neuropathy. Blood glucose levels were significantly elevated compared with control rats one week after injection of STZ. Thereafter, hyperglycaemia was maintained throughout the experimental period (Table 1). Diabetic rats had polyphagia and polyposia in the general observation, but showed significantly lower body weight than control rats one week after injection of STZ. The rate of increase in the body weight of diabetic rats was much slower than that of control rats throughout the experimental period (Fig. 1).
Table 1. Time-course Changes in Glycemia

\begin{tabular}{lcll}
\hline Group & pre & 1 week & 8 weeks \\
\hline control (mg/dL) & $108 \pm 14$ & $122 \pm 4$ & $133 \pm 23$ \\
STZ (mg/dL) & $114 \pm 12$ & $499 \pm 53^{* * *}$ & $484 \pm 46^{* * *}$ \\
\hline
\end{tabular}

Blood glucose levels were significantly elevated 1 week after STZ injection compared to the control, and persisted for the 8 weeks of testing. Data are the means \pm S.D. ${ }^{* * *}: \mathrm{P}<0.001$; Significant difference from control.

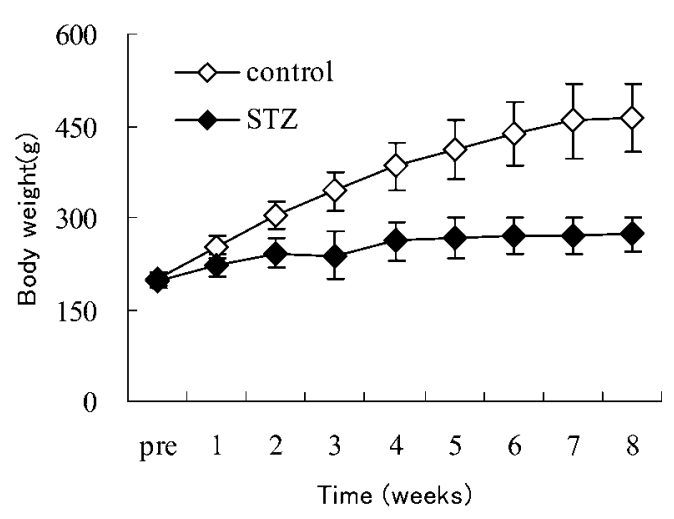

Fig. 1. Time-course change in body weights of STZ and control rats. Each point is the mean \pm S.D. Differences between STZ and control rats were significant $(\mathrm{P}<0.05)$ from 1 week after treatment.

\section{Histological results in the pancreas of STZ-induced diabetic rats}

STZ-induced diabetic rats showed atrophy of the pancreatic islets and particularly marked atrophy of beta cells, which caused severely decreased expression of insulin, according to immunohistochemical examination. Moreover, the exocrine secretory tissue presented a dark color in comparison with the control group (Fig. 2).

\section{Mechanical nociceptive thresholds}

The mechanical threshold was monitored after injection of STZ. The force-withdrawal-threshold was significantly lower in diabetic rats than in control rats. This mechanical hypersensitivity developed 2 weeks after STZ injection and persisted for the 8 weeks of testing (Fig. 3). It was confirmed that STZ-induced diabetic rats suffer from hyperalgesia.

\section{Effect of capsaicin cream on the mechanical hyperalgesia in diabetic rats}

When the STZ group received cream base, hyperalgesia was significant compared with the control group, but there was no difference between the groups when they both received capsaicin cream. Moreover, in terms relative to the threshold before the application, expressed as $100 \%$, the STZ group was at the same level as the control group receiving the cream base application, but the sharp pain threshold was significantly increased by the application of 

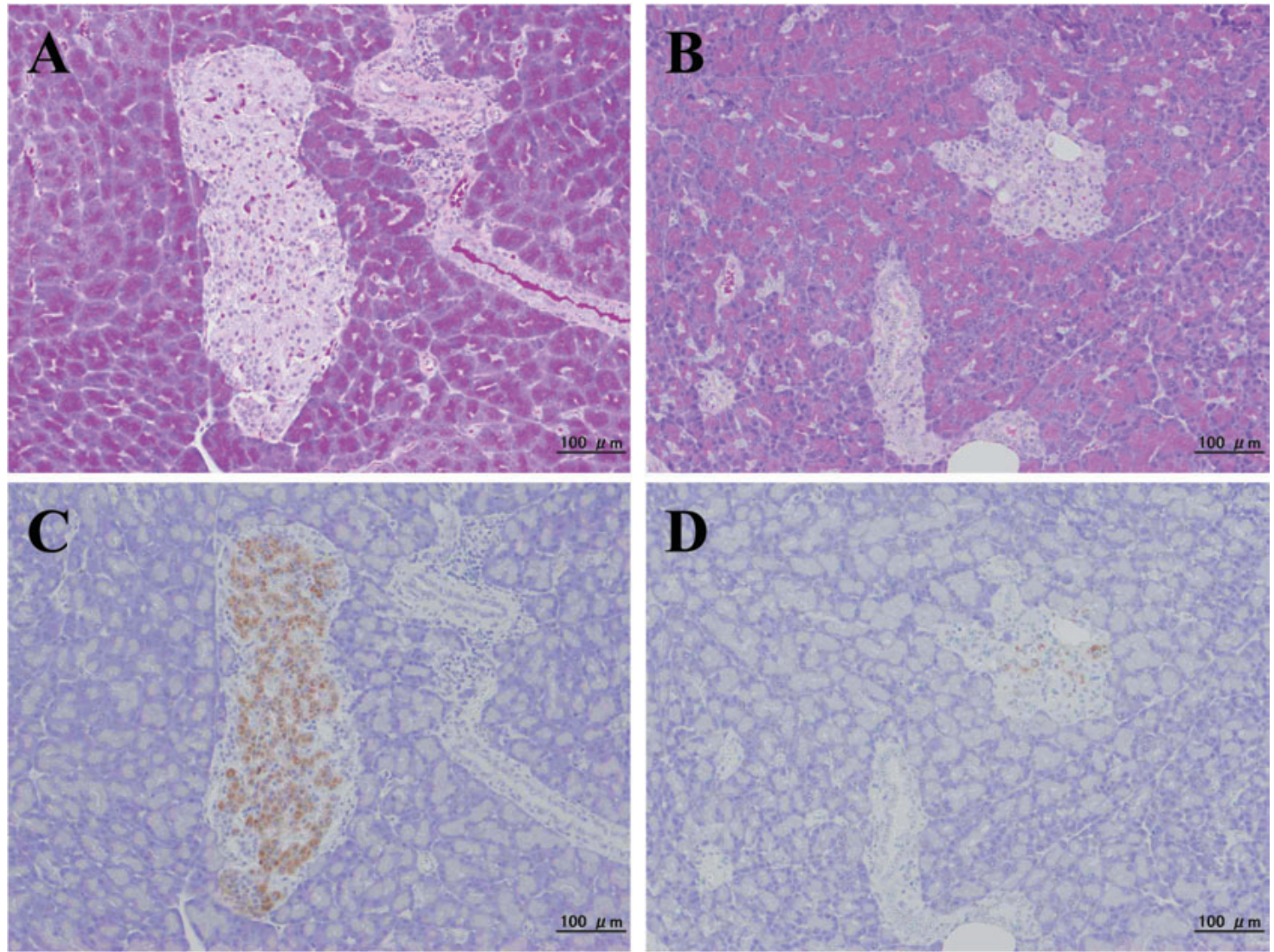

Fig. 2. Light microscopic changes of pancreas after treatment. The pancreas islets of STZ rats (B) showed marked atrophy and dark exocrine secretion tissue in comparison with control (A) by HE staining. Also STZ rats (D) had severely decreased expression of insulin by immnunohistochemical staining in comparison with control (C). Scale bar $=100 \mu \mathrm{m}$.

$0.1 \%$ capsaicin cream compared with the control group (Table 2).

Immunohistochemical results in the DRG of STZinduced diabetic rats

VR1, NF200, and the VR1: NF200 positive ratios (data not shown) in DRG (L4, 5, and 6) were equal in the control group and the STZ group (Fig. 4).

Histochemical results in the hind paw skin of STZinduced diabetic rats

In HE specimens, the STZ group showed no remarkable differences, except for cutaneous thickness compared with the control group, and no toxicological change by the single application of $0.1 \%$ capsaicin cream was observed in either of the groups (Fig. 5).

The immunohistochemical test of the cutaneous nerve showed no significant difference in the PGP 9.5-positive areas between the STZ group and the control group. However, positive areas of NF200 were significantly increased in the STZ group (Table 3, Fig. 6).

\section{Discussion}

We confirmed the analgesic effect of capsaicin in a rat

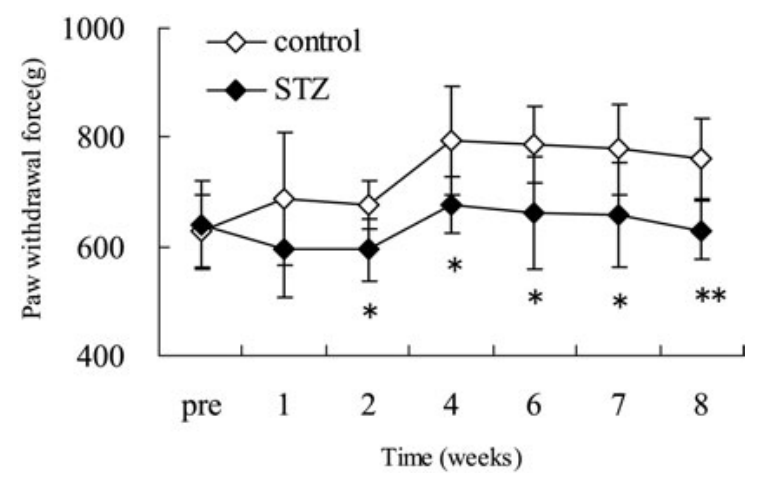

Fig. 3. Time-course of change in mechanical nociceptive threshold. The mechanical nociceptive threshold of STZ rats decreased at 2 weeks after treatment and this decrease persisted for the 8 weeks of testing. Each point is the mean \pm S.D. Differences between STZ and control rats were significant $(*: \mathrm{P}<0.05$, $* *: \mathrm{P}<0.01)$.

model of STZ painful diabetic neuropathy and examined its neurohistological relevance to the effect.

Hyperglycaemia had already occurred by one week after intravenous injection of STZ. Although the STZ rats had polyphagia and polyposia, their body weight was little 
Table 2. Effect of Capsaicin Cream on Mechanical Nociceptive Thresholds

\begin{tabular}{|c|c|c|c|c|c|}
\hline \multicolumn{2}{|l|}{ Group } & \multicolumn{2}{|c|}{ Cream base } & \multicolumn{2}{|c|}{$0.1 \%$ Capsaicin cream } \\
\hline & & pre & post & pre & post \\
\hline \multirow[t]{2}{*}{ control } & $\mathrm{g}^{\mathrm{a}}$ & $778 \pm 83$ & $840 \pm 88$ & $761 \pm 73$ & $746 \pm 101$ \\
\hline & $\% \mathrm{~b}$ & 100.0 & $108.9 \pm 16.1$ & 100.0 & $97.9 \pm 7.3$ \\
\hline \multirow[t]{2}{*}{ STZ } & $\mathrm{g}^{\mathrm{a}}$ & $658 \pm 97 *$ & $728 \pm 80^{*}$ & $629 \pm 55^{* *}$ & $702 \pm 64$ \\
\hline & $\% \mathrm{~b}$ & 100.0 & $111.6 \pm 11.2$ & 100.0 & $111.9 \pm 9.7 *$ \\
\hline
\end{tabular}

The mechanical nociceptive threshold of the STZ rats did not change when cream base was applied, but the threshold elevated in comparison to the control when the $0.1 \%$ capsaicin cream was applied. a: absolute value $(\mathrm{g})$, b: relative value $(\%$, relative to the pre-application value of $100 \%$ ). Data are means \pm S.D. *: $\mathrm{P}<0.05,{ }^{* *}: \mathrm{P}<0.01$; Significant difference from control.
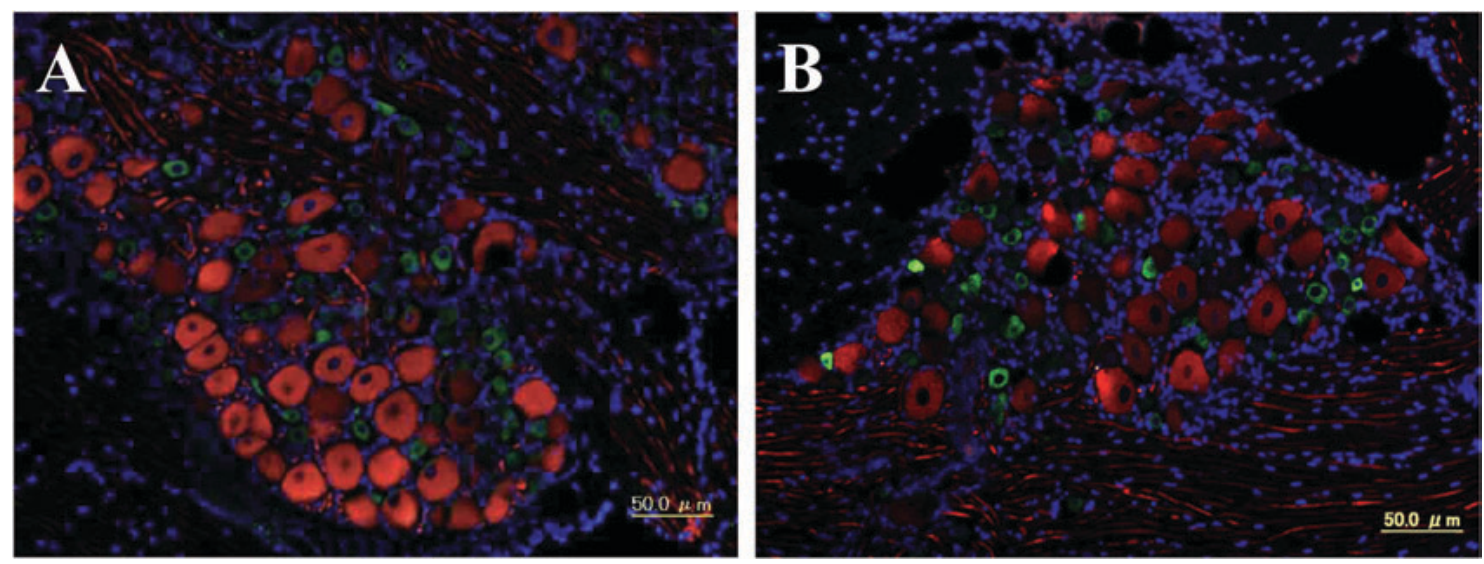

Fig. 4. Immunohistochemical staining for VR1 and NF200 of DRG neurons. The DRG neurons of control (A) and STZ (B) rats were labeled VR1 (green), NF200 (red), and DAPI (blue). Scale bar $=50 \mu \mathrm{m}$.

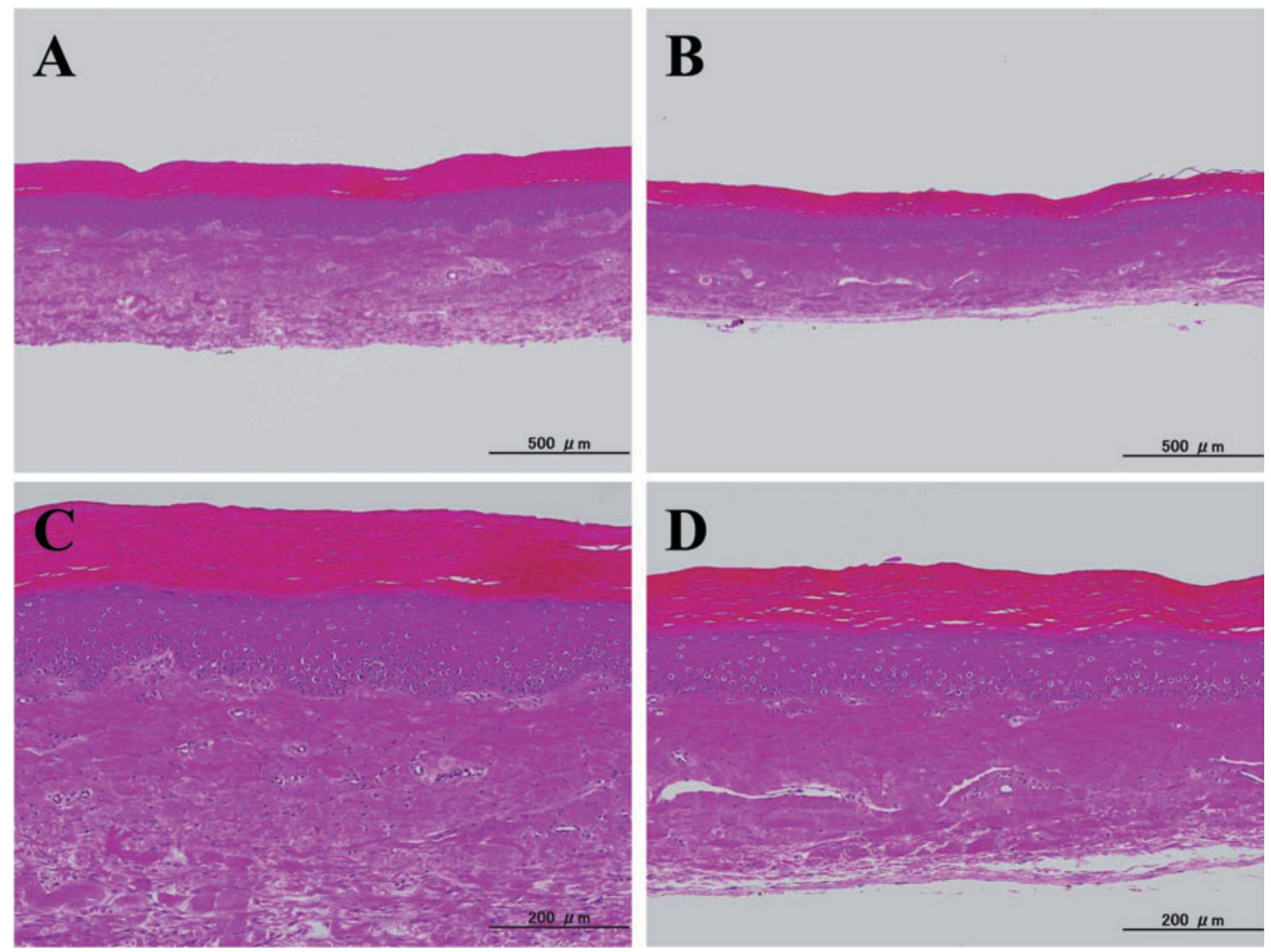

Fig. 5. Light microscopic changes of hind paw after treatment. The hind paw of control (A, C) and STZ (B, D) rats did not show toxicological changes. HE staining, A, B: lower magnification (scale bar $=500 \mu \mathrm{m}$ ), C, D: higher magnification (scale bar $=200 \mu \mathrm{m}$ ). 

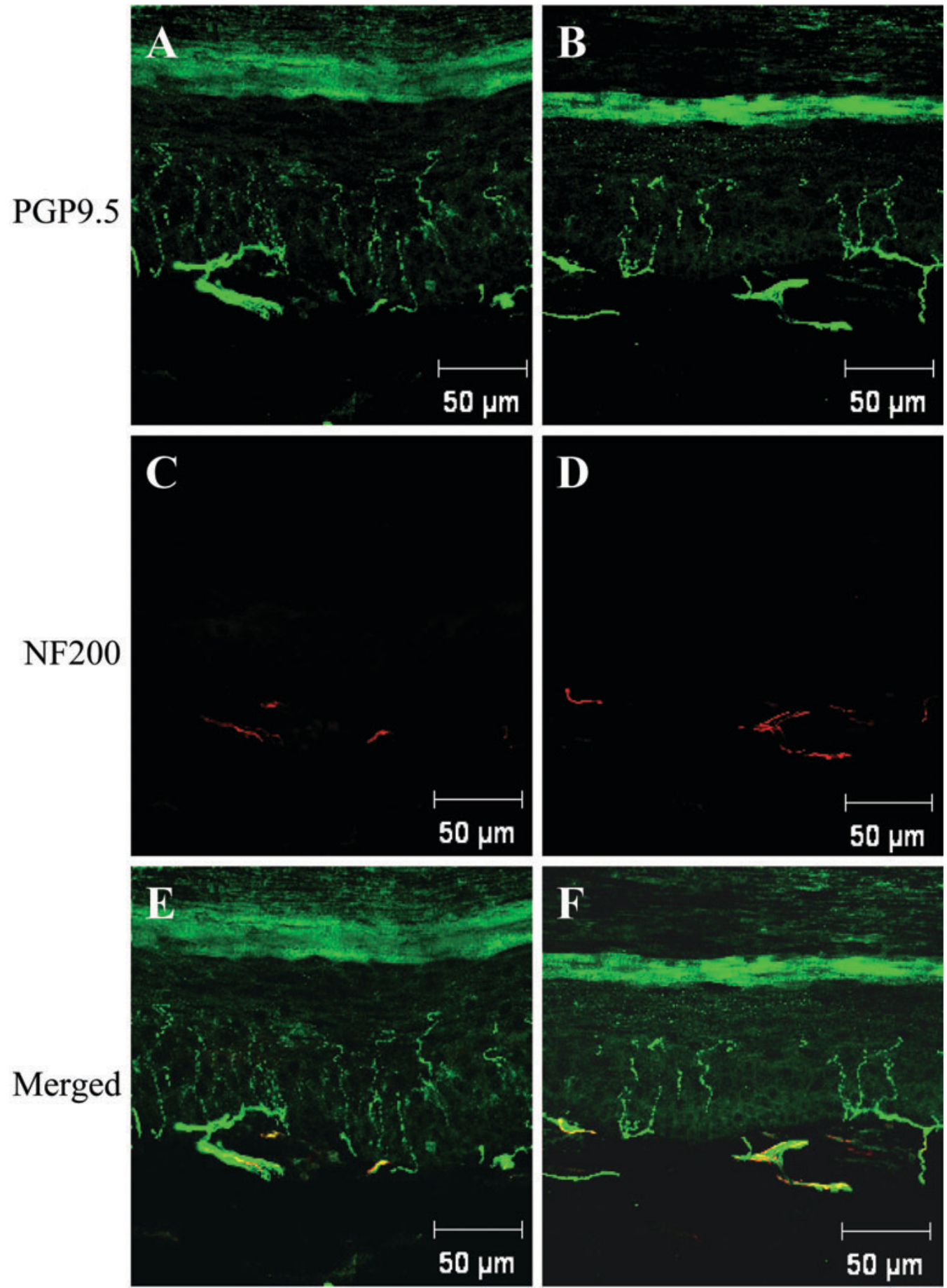

Fig. 6. Confocal microscopy of double immunoflourescence staining of PGP9.5 (green) and NF200 (red). In the merged images, colocalization is represented in yellow. A, C, E: control rats, B, D, F: STZ rats. Scale bar $=50 \mu \mathrm{m}$.

increased. These findings are in accord with much previous data $^{2,5,6,19}$. We confirmed STZ insulin-dependent diabetes from the results of the atrophy of the pancreatic islets and decreased insulin expression by histochemical examination of the pancreas. In addition, the dark exocrine cells seemed to be caused by polyphagia and polyposia.

Mechanical hyperalgesia occurred two weeks after STZ injection. There are some reports suggesting the underlying mechanisms of hyperalgesia in this model ${ }^{13,20-23}$. In one of the mechanisms, expression of VR1 are observed in capsaicin-insensitive A-fiber DRG neurons of STZ diabetic mice suggested as a cause of the hyperalgesia. Although we confirmed VR1 was expressed, we were not able to observe the expression of VR1 in A-fibers of the STZ rats. This was possibly to be attributable to the difference between the specimen preparation methods we used (AMeX fixation and 
Table 3. Immunohistochemical Areas of Nerve Fibers in the Hind Paw

\begin{tabular}{lccc}
\hline Group & PGP9.5 $\left(\mu \mathrm{m}^{2}\right)$ & NF200 $\left(\mu \mathrm{m}^{2}\right)$ & NF200/PGP9.5 ratio(\%) \\
\hline control & $795.4 \pm 187.6$ & $99.1 \pm 39.0$ & $12.5 \pm 4.1$ \\
STZ & $779.6 \pm 261.6$ & $199.8 \pm 85.6^{*}$ & $27.5 \pm 11.1^{*}$ \\
\hline
\end{tabular}

STZ rats showed no difference in PGP9.5 immunoreactive area, but had a significantly increased NF200 immunoreactive area and NF200/PGP9.5 ratio in comparison with the control at 8 weeks after treatment. Data are means \pm S.D. *: $\mathrm{P}<0.05$; Significant difference from control.

paraffin section versus paraformaldehyde fixation and frozen section), or the VR1 antibodies we used (manufacturers). We will examine VR1 expression of Afiber DRG neurons more closely in the future.

On the other hand, the percentage of A-fibers increased in the cutaneous nerve. Because A-fibers have mechanical nociceptors, it was expected that hyperalgesia by mechanical nociceptive stimuli would be observed in this experiment. However, it is essential that the immunohistological examination we conducted should be performed not only by light microscope but also by electron microscope for the clarification of the relationship between hyperalgesia and increased A-fibers in the cutaneous nerve.

The hyperalgesia in the STZ rats disappeared after capsaicin cream was applied. The analgesic effect of capsaicin was reported to be effective in mice with hyperalgesia ${ }^{13}$, and was also confirmed in rats in this study. The threshold did not decrease when the capsaicin cream was applied to normal animals, suggesting that sensitivity to capsaicin develops in STZ rats.

We applied $0.1 \%$ capsaicin cream once in the present study. It is common that capsaicin cream is applied repeatedly in clinical practice, and there is a report suggesting that analgesic effect was observed by repeated application of capsaicin ${ }^{24}$. It was suggested in that report, that depletion of neuropeptide substance $\mathrm{P}$ and calcitonin gene-related peptide (CGRP) released from C-fibers caused the effect, but desensitization through VR1 is considered to be the main action mechanism of the analgesic effect in the recent reports ${ }^{9-12,25,26}$. In addition, there are reports suggesting that the analgesic effect occurs because the epidermal nerve fibers of the parts to which capsaicin was applied, degenerate from a morphologic point of view ${ }^{15,16,27}$. In the present study, it was not clear whether the analgesic effect was caused by degeneration of the nerve fiber or by desensitization after the single application of capsaicin. However, no report has observed a morphological change of nerve fibers after a single application of capsaicin; thus, the change may be considered due to the desensitization. It seems likely that desensitization is involved in a continuum of changes in the primary sensory neurons, from physiological to morphological, that are dependent on capsaicin dose, route of administration, and susceptibility of the neuron, and its processes. Furthermore, a change of Afibers was suggested in STZ rats, but the relationships between the morphologic change and the desensitization are uncertain, so this is an issue which needs further investigation.

In conclusion, a single systemic injection of STZ to rats induced painful diabetic neuropathy, and it was suggested that hyperalgesia resulted from an increase in A-fibers of the peripheral nerve. In addition, the hyperalgesia was reduced by single application of capsaicin cream, but the mechanism for the reduction was not revealed by histological changes. It seems that increased peripheral A-fibers are involved in the antihyperalgesic effect of capsaicin cream. Capsaicin cream may become an effective therapeutic agent if the mechanism for the antihyperalgesic effect can be clarified and the relationship between its analgesic effects and patients' neuropathies can be determined.

Acknowledgements: We gratefully acknowledge Drs. M. Watanabe, Anatomy, Osaka Medical College for kindly lending the laser-scanning confocal microscope and for helpful advice on our study. We also thank Mrs. M. Kawashima for her cooperation.

\section{References}

1. Rakieten N, Rakieten ML, and Nadkarni MR. Studies on the diabetogenic action of streptozotocin. Cancer Chemother Rep. 29: 91-98. 1963.

2. Kanarek RB and Ho L. Patterns of nutrient selection in rats with streptozotocin-induced diabetes. Physiol Behav. 32: 639-645. 1984.

3. Ahlgren SC and Levine JD. Mechanical hyperalgesia in streptozotocin-diabetic rats. Neuroscience. 52: 1049-1055. 1993.

4. Malcangio M and Tomlinson DR. A pharmacologic analysis of mechanical hyperalgesia in streptozotocin/diabetic rats. Pain. 76: 1998. 151-157.

5. Fox A, Eastwood C, Gentry C, Manning D, and Urban L. Critical evaluation of the streptozotocin model of painful diabetic neuropathy in the rat. Pain. 81: 307-316. 1999.

6. Aubel B, Kayser V, Mauborgne A, Farré A, Hamon M, and Bourgoin S. Antihyperalgesic effects of cizolirtine in diabetic rats: behavioral and biochemical studies. Pain. 110: 22-32. 2004.

7. Low PA, Opfer-Gehrking TL, Dyck PJ, Litchy WJ, and O'Brien PC. Double-blind, placebo-controlled study of the application of capsaicin cream in chronic distal painful polyneuropathy. Pain. 62: 163-168.1995.

8. The Capsaicin Study Group. Treatment of painful diabetic neuropathy with topical capsaicin. A multicenter, doubleblind, vehicle-controlled study. Arch Intern Med. 151: 2225-2229. 1991.

9. Caterina MJ, Schumacher MA, Tominaga M, Rosen TA, Levine JD, and Julius D. The capsaicin receptor: a heatactivated ion channel in the pain pathway. Nature. 389: 816824. 1997.

10. Tominaga $M$, Caterina MJ, Malmberg AB, Rosen TA, Gilbert H, Skinner K, Raumann BE, Basbaum AI, and Julius D. The cloned capsaicin receptor integrates multiple painproducing stimuli. Neuron. 21: 531-543. 1998.

11. Tominaga $M$, Wada $M$, and Masu M. Potentiation of 
capsaicin receptor activity by metabotropic ATP receptors as a possible mechanism for ATP-evoked pain and hyperalgesia. Proc Natl Acad Sci. 98: 6951-6956. 2001.

12. Garcia-Martinez C, Humet M, Planells-Cases R, Gomis A, Caprini M, Viana F, De La Pena E, Sanchez-Baeze F, Carbonell T, De Felipe C, Pérez-Paya E, Belmonte C, Messequer A, and Ferrer-Montiel A. Attenuation of thermal nociception and hyperalgesia by VR1 blockers. Proc Natl Acad Sci. 99: 2374-2379. 2002.

13. Rashid $\mathrm{MH}$, Inoue $\mathrm{M}$, Bakoshi S, and Ueda H. Increased expression of vanilloid receptor 1 on myelinated primary afferent neurons contributes to the antihyperalgesic effect of capsaicin cream in diabetic neuropathic pain in mice. $J$ Pharmacol Exp Ther. 306: 709-717. 2003.

14. Nolano M, Simone DA, Wendelschafer-Crabb G, Johnson T, Hazen E, and Kennedy WR. Topical capsaicin in humans: parallel loss of epidermal nerve fibers and pain sensation. Pain. 81: 135-145. 1999.

15. Khalili N, Wendelschafer-Crabb G, Kennedy WR, and Simone DA. Influence of thermode size for detecting heat pain dysfunction in a capsaicin model of epidermal nerve fiber loss. Pain. 91: 241-250. 2001.

16. Martinez-Nieves B and Dunbar JC. Vascular dilatatory responses to sodium nitroprusside (SNP) and $\alpha$-adrenergic antagonism in female and male normal and diabetic rats. Proc Soc Exp Biol Med. 222: 90-98. 1999.

17. Takahashi Y and Nakajima Y. Dermatomes in the rat limbs as determined by antidromic stimulation of sensory C-fibers in spinal nerves. Pain. 67: 197-202. 1996.

18. Sato Y, Mukai K, Watanabe S, Goto M, and Shimosato Y. The AMeX method. A simplified technique of tissue processing and paraffin embedding with improved preservation of antigens for immunostaining. Am J Pathol. 125: 431-435. 1986.

19. Saito TR, Tatsuno T, Takeda A, Hashimoto H, Suzuki M,
Terada M, Aoki-Komori S, Tanaka M, Koranyi L, and Nagy GM. Effects of i.c.v. administration of Leptin on copulatory and ingestive behavior in STZ-induced diabetic male rats. Exp Anim. 53: 445-451. 2004.

20. Bölcskei K, Helyes Z, Szabó A, Sándor K, Elekes K, Németh J, Almási R, Pintér E, Petho G, and Szolcsányi J. Investigation of the role of TRPV1 receptors in acute and chronic nociceptive processes using gene-deficient mice. Pain. 117: 368-376. 2005.

21. Yusaf SP, Goodman J, Gonzalez IM, Bramwell S, Pinnock RD, Dixon AK, and Lee K. Streptozocin-induced neuropathy is associated with altered expression of voltagegated calcium channel subunit mRNAs in rat dorsal root ganglion neurones. Biochem Biophys Res Commun. 289: 402-406. 2001.

22. Hong S, Morrow TJ, Paulson PE, Isom LL, and Wiley JW. Early painful diabetic neuropathy is associated with differential changes in tetrodotoxin-sensitive and -resistant sodium channels in dorsal root ganglion neurons in the rat. $\mathrm{J}$ Biol Chem. 279: 29341-29350. 2004.

23. Hong S and Wiley JW. Early painful diabetic neuropathy is associated with differential changes in the expression and function of vanilloid receptor 1. J Biol Chem. 280: 618-627. 2005.

24. Fuchs PN, Raja SN, and Meyer RA. Topical capsaicin for the treatment of neuropathic pain. Analgesia. 5: 1-8. 2000.

25. Szallasi A and Blumberg PM. Vanilloid (capsaicin) receptors and mechanisms. Pharm Rev. 51: 159-211.1999.

26. Sawynok J. Topical and peripherally acting analgesics. Pharm Rev. 55: 1-20. 2003.

27. Simone DA, Nolano M, Johnson T, Wendelschafer-Crabb $\mathrm{G}$, and Kennedy WR. Intradermal injection of capsaicin in humans produces degeneration and subsequent reinnervation of epidermal nerve fibers: correlation with sensory function. J Neuroscience. 18: 8947-8959. 1998. 